\title{
The fourth year of Internal and Emergency Medicine
}

\section{Gian Franco Gensini}

Published online: 20 December 2008

(c) SIMI 2008

Internal and Emergency Medicine, the Official Journal of the Italian Society of Internal Medicine (SIMI), has been steadily growing over the last few years; the time has come to present our readers the editorial activities and plans for 2009.

In February 2007, the electronic manuscript submission system Editorial Manager was introduced to guarantee the authors a prompt turnaround time for the review process and a rapid electronic publication. Since its introduction, the number of manuscripts submitted has significantly increased by $54 \%$ in 2008 over 2007. The use of the Editorial Manager has furthermore led to a decrease in the review time: the average time from submission to acceptance decreased by $33 \%$; the average time from submission to rejection decreased by $25 \%$. The peer-review process has become more and more selective; this seems to have influenced the quality of accepted papers. For these reasons, the Editorial Board of the journal decided to increase the frequency of the journal: Internal and Emergency Medicine will have 6 issues per year instead of 4 starting in January 2009.

To guarantee a maximum exposure and visibility of the journal, all manuscripts are published not only in print but also in the electronic version on the online platform SpringerLink. The OnlineFirst service lets users gain access to peer reviewed articles weeks before distribution of the printed journal. Another important fact is that these articles are not only searchable but also citable by their digital object identifier (DOI). The time it takes for critical discoveries to reach the research community is hence significantly reduced.

Additionally, the journal has been included in the Abstracting/Indexing Institutes Google Scholar, InPharma, Medline/PubMed and Scopus and is thereby receiving attention of a broader audience.

Furthermore I am very glad to announce that I have been assigned by the Italian Society of Internal Medicine to work for another year together with all the Members of the Editorial Board and of the Operative Group to reach our main goal: to obtain, hopefully soon, an impact factor for the Journal.

We really hope you will continue to support our Journal and to participate in this new challenge.
G. F. Gensini $(\bowtie)$

Editor-in-Chief, Internal and Emergency Medicine, Department of Critical Care Medicine and Surgery, University of Florence and Azienda Ospedaliero-Universitaria Careggi, Viale Morgagni 85, 50134 Florence, Italy e-mail: g.gensini@dac.unifi.it 